\title{
Thermal Properties of Light Tensor Mesons via QCD Sum Rules
}

\author{
K. Azizi, ${ }^{1}$ A. Türkan, ${ }^{2}$ E. Veli Veliev, ${ }^{2}$ and H. Sundu' ${ }^{2}$ \\ ${ }^{1}$ Department of Physics, Faculty of Arts and Sciences, Doğuş University, Acibadem, Kadikoy, 34722 Istanbul, Turkey \\ ${ }^{2}$ Department of Physics, Kocaeli University, 41380 Izmit, Turkey \\ Correspondence should be addressed to K. Azizi; kazizi@dogus.edu.tr
}

Received 27 August 2014; Revised 20 February 2015; Accepted 25 February 2015

Academic Editor: Kadayam S. Viswanathan

Copyright (C) $2015 \mathrm{~K}$. Azizi et al. This is an open access article distributed under the Creative Commons Attribution License, which permits unrestricted use, distribution, and reproduction in any medium, provided the original work is properly cited. The publication of this article was funded by $\mathrm{SCOAP}^{3}$.

The thermal properties of $f_{2}(1270), a_{2}(1320)$, and $K_{2}^{*}(1430)$ light tensor mesons are investigated in the framework of QCD sum rules at finite temperature. In particular, the masses and decay constants of the light tensor mesons are calculated taking into account the new operators appearing at finite temperature. The numerical results show that, at the point at which the temperature-dependent continuum threshold vanishes, the decay constants decrease with amount of (70-85)\% compared to their vacuum values, while the masses diminish about (60-72)\% depending on the kinds of the mesons under consideration. The results obtained at zero temperature are in good consistency with the experimental data as well as the existing theoretical predictions.

\section{Introduction}

The study of strong interaction at low energies is one of the most important problems of the high energy physics. This can play a crucial role in exploring the structure of mesons, baryons, and vacuum properties of strong interaction. The tensor particles can provide a different perspective for understanding the low energy QCD dynamics. In recent decades, great efforts have been made both experimentally and theoretically to investigate the tensor particles in order to understand their nature and internal structure.

The investigation of hadronic properties at finite baryon density and temperature in QCD also plays an essential role in interpretation of the results of heavy-ion collision experiments and obtaining the QCD phase diagram. The Compressed Baryonic Matter (CBM) experiment of the FAIR project at GSI is important for understanding the way of Chiral symmetry realization in the low energy region and, consequently, the confinement of QCD. According to thermal QCD, the hadronic matter undergoes quark gluon-plasma phase at a critical temperature. These kinds of phase may exist in the neutron stars and early universe. Hence, calculation of the parameters of hadrons via thermal QCD may provide us with useful information on these subjects.
The restoration of Chiral symmetry at high temperature requires the medium modifications of hadronic parameters [1]. There are many nonperturbative approaches to hadron physics. The QCD sum rule method $[2,3]$ is one of the most attractive and applicable tools in this respect. In this approach, hadrons are represented by their interpolating quark currents and the correlation function of these currents is calculated using the operator product expansion (OPE). The thermal version of this approach is based on some basic assumptions so that the Wilson expansion and the quarkhadron duality approximation remain valid, but the vacuum condensates are replaced by their thermal expectation values [4]. At finite temperature, the Lorentz invariance is broken and, due to the residual $O(3)$ symmetry, some new operators appear in the Wilson expansion [5-7]. These operators are expressed in terms of the four-vector velocity of the medium and the energy momentum tensor. There are numerous works in the literature on the medium modifications of parameters of (pseudo)scalar and (axial)vector mesons using different theoretical approaches, for example, Chiral model [8], coupled channel approach $[9,10]$, and QCD sum rules $[5,6,11-17]$. Recently, we applied this method to calculate some hadronic parameters related to the charmed $D_{2}^{*}(2460)$ and charmed-strange $D_{s 2}^{*}(2573)$ tensor [18] mesons. 
In the present work we investigate the properties of light $a_{2}(1320), f_{2}(1270)$, and $K_{2}^{*}(1430)$ tensor mesons in the framework of QCD sum rules at finite temperature. We also compare the results obtained at zero temperature with the predictions of some previous studies on the parameters of the same mesons in vacuum [19-21].

The present paper is organized as follows. In the next section, considering the new operators raised at finite temperature, we evaluate the corresponding thermal correlation function to obtain the QCD sum rules for the parameters of the mesons under consideration. The last section is devoted to the numerical analysis of the sum rules obtained as well as investigation of the sensitivity of the masses and decay constants of the light tensor mesons on temperature.

\section{Thermal QCD Sum Rules for Masses and Decay Constants of Light Tensor Mesons}

In this section we present the basics of the thermal QCD sum rules and apply this method to some light tensor mesons like $f_{2}(1270), a_{2}(1320)$, and $K_{2}^{*}(1430)$ to compute their mass and decay constant. The starting point is to consider the following thermal correlation function:

$$
\Pi_{\mu \nu, \alpha \beta}(q, T)=i \int d^{4} x e^{i q \cdot(x-y)} \operatorname{Tr}\left\{\rho \mathscr{T}\left[J_{\mu \nu}(x) \bar{J}_{\alpha \beta}(y)\right]\right\},
$$

where $\rho=e^{-\beta H} / \operatorname{Tr}\left(e^{-\beta H}\right)$ is the thermal density matrix of QCD, $\beta=1 / T$ with $T$ being temperature, $H$ is the QCD Hamiltonian, $\mathscr{T}$ indicates the time ordered product, and $J_{\mu \nu}$ is the interpolating current of tensor mesons. The interpolating fields for these mesons can be written as

$$
\begin{aligned}
& J_{\mu \nu}^{K_{2}^{*}}(x)=\frac{i}{2}\left[\bar{s}(x) \gamma_{\mu} \stackrel{\leftrightarrow}{\mathscr{D}}_{\nu}(x) d(x)+\bar{s}(x) \gamma_{\nu} \stackrel{\leftrightarrow}{\mathscr{D}}_{\mu}(x) d(x)\right], \\
& J_{\mu \nu}^{f_{2}}(x)=\frac{i}{2 \sqrt{2}}\left[\bar{u}(x) \gamma_{\mu} \stackrel{\leftrightarrow}{D}_{\nu}(x) u(x)+\bar{u}(x) \gamma_{\nu} \stackrel{\leftrightarrow}{\mathscr{D}}_{\mu}(x) u(x)\right. \\
& +\bar{d}(x) \gamma_{\mu} \stackrel{\leftrightarrow}{\mathscr{D}}_{\nu}(x) d(x) \\
& \left.+\bar{d}(x) \gamma_{\nu} \stackrel{\leftrightarrow}{\mathscr{D}}_{\mu}(x) d(x)\right], \\
& J_{\mu \nu}^{a_{2}}(x)=\frac{i}{2 \sqrt{2}}\left[\bar{u}(x) \gamma_{\mu} \stackrel{\leftrightarrow}{\mathscr{D}}_{\nu}(x) u(x)+\bar{u}(x) \gamma_{\nu} \stackrel{\leftrightarrow}{\mathscr{D}}_{\mu}(x) u(x)\right. \\
& -\bar{d}(x) \gamma_{\mu} \stackrel{\leftrightarrow}{\mathscr{D}}_{\nu}(x) d(x) \\
& \left.-\bar{d}(x) \gamma_{\nu} \stackrel{\leftrightarrow}{D}_{\mu}(x) d(x)\right],
\end{aligned}
$$

where $\stackrel{\leftrightarrow}{D}_{\mu}(x)$ denotes the derivative with respect to four- $x$ simultaneously acting on left and right. It is given as

$$
\stackrel{\leftrightarrow}{\mathscr{D}}_{\mu}(x)=\frac{1}{2}\left[\overrightarrow{\mathscr{D}}_{\mu}(x)-\overleftarrow{\mathscr{D}}_{\mu}(x)\right]
$$

where

$$
\begin{aligned}
& \vec{D}_{\mu}(x)=\vec{\partial}_{\mu}(x)-i \frac{g}{2} \lambda^{a} A_{\mu}^{a}(x), \\
& \mathscr{D}_{\mu}(x)=\overleftarrow{\partial}_{\mu}(x)+i \frac{g}{2} \lambda^{a} A_{\mu}^{a}(x),
\end{aligned}
$$

with $\lambda^{a}(a=1,8)$ and $A_{\mu}^{a}(x)$ being the Gell-Mann matrices and external gluon fields, respectively. The currents contain derivatives with respect to the space-time; hence, we consider the two currents at points $x$ and $y$ in (1), but, for simplicity, we will set $y=0$ after applying derivative with respect to $y$.

It is well known that, in thermal QCD sum rule approach, the thermal correlation function can be calculated in two different ways. Firstly, it is calculated in terms of hadronic parameters such as masses and decay constants. Secondly, it is calculated in terms of the QCD parameters such as quark masses, quark condensates, and quark-gluon coupling constants. The coefficients of sufficient structures from both representations of the same correlation function are then equated to find the sum rules for the physical quantities under consideration. We apply Borel transformation and continuum subtraction to both sides of the sum rules in order to further suppress the contributions of the higher states and continuum.

Let us focus on the calculation of the hadronic side of the correlation function. For this aim we insert a complete set of intermediate physical states having the same quantum numbers as the interpolating current into (1). After performing integral over four- $x$ and setting $y=0$, we get

$$
\begin{aligned}
\Pi_{\mu \nu, \alpha \beta}(q, T) & \\
= & \frac{\left\langle 0\left|J_{\mu \nu}(0)\right| K_{2}^{*}\left(f_{2}\right)\left(a_{2}\right)\right\rangle\left\langle K_{2}^{*}\left(f_{2}\right)\left(a_{2}\right)\left|\bar{J}_{\alpha \beta}(0)\right| 0\right\rangle}{m_{K_{2}^{*}\left(f_{2}\right)\left(a_{2}\right)}^{2}-q^{2}} \\
& +\cdots,
\end{aligned}
$$

where dots indicate the contributions of the higher and continuum states. The matrix element $\left\langle 0\left|J_{\mu \nu}(0)\right| K_{2}^{*}\left(f_{2}\right)\left(a_{2}\right)\right\rangle$ creating the tensor mesons from vacuum can be written in terms of the decay constant, $f_{K_{2}^{*}\left(f_{2}\right)\left(a_{2}\right)}$, as

$$
\left\langle 0\left|J_{\mu \nu}(0)\right| K_{2}^{*}\left(f_{2}\right)\left(a_{2}\right)\right\rangle=f_{K_{2}^{*}\left(f_{2}\right)\left(a_{2}\right)} m_{K_{2}^{*}\left(f_{2}\right)\left(a_{2}\right)}^{3} \varepsilon_{\mu \nu}^{(\lambda)},
$$

where $\varepsilon_{\mu \nu}^{(\lambda)}$ is the polarization tensor. Using (6) in (5), the final expression of the physical side is obtained as

$$
\begin{aligned}
\Pi_{\mu \nu, \alpha \beta}(q, T) & \\
= & \frac{f_{K_{2}^{*}\left(f_{2}\right)\left(a_{2}\right)}^{2} m_{K_{2}^{*}\left(f_{2}\right)\left(a_{2}\right)}^{6}\left\{\frac{1}{2}\left(g_{\mu \alpha} g_{\nu \beta}+g_{\mu \beta} g_{\nu \alpha}\right)\right\}}{K_{2}^{*}\left(f_{2}\right)\left(a_{2}\right)}-q^{2} \\
& + \text { other structures }+\cdots,
\end{aligned}
$$


where the only structure that we will use in our calculations has been shown explicitly. To obtain the above expression, we have used the summation over polarization tensors as

$$
\sum_{\lambda} \varepsilon_{\mu \nu}^{(\lambda)} \varepsilon_{\alpha \beta}^{*(\lambda)}=\frac{1}{2} T_{\mu \alpha} T_{\nu \beta}+\frac{1}{2} T_{\mu \beta} T_{\nu \alpha}-\frac{1}{3} T_{\mu \nu} T_{\alpha \beta},
$$

where

$$
T_{\mu \nu}=-g_{\mu \nu}+\frac{q_{\mu} q_{\nu}}{m_{K_{2}^{*}\left(f_{2}\right)\left(a_{2}\right)}^{2}} .
$$

Now we concentrate on the OPE side of the thermal correlation function. In OPE representation, the coefficient of the selected structure can be separated into perturbative and nonperturbative parts:

$$
\Pi(q, T)=\Pi^{\text {pert }}(q, T)+\Pi^{\text {non-pert }}(q, T) .
$$

The perturbative or short-distance contributions are calculated using the perturbation theory. This part in spectral representation is written as

$$
\Pi^{\text {pert }}(q, T)=\int d s \frac{\rho(s)}{s-q^{2}},
$$

where $\rho(s)$ is the spectral density and it is given by the imaginary part of the correlation function; that is,

$$
\rho(s)=\frac{1}{\pi} \operatorname{Im}\left[\Pi^{\text {pert }}(s, T)\right] .
$$

The nonperturbative or long-distance contributions are represented in terms of thermal expectation values of the quark and gluon condensates as well as thermal average of the energy density. Our main task in the following is to calculate the spectral density as well as the nonperturbative contributions. For this aim, we use the explicit forms of the interpolating currents for the tensor mesons in (1). After contracting out all quark fields using Wick's theorem, we get

$$
\begin{aligned}
& \Pi_{\mu \nu, \alpha \beta}^{K_{2}^{*}}(q, T) \\
& =-\frac{i}{4} \int d^{4} x e^{i q \cdot(x-y)} \\
& \cdot\left\{\operatorname{Tr}\left[S_{s}(y-x) \gamma_{\mu} \stackrel{\leftrightarrow}{D}_{\nu}(x) \stackrel{\leftrightarrow}{\mathscr{D}}_{\beta}(y) S_{d}(x-y) \gamma_{\alpha}\right]\right. \\
& +[\beta \longleftrightarrow \alpha]+[\nu \longleftrightarrow \mu] \\
& +[\beta \longleftrightarrow \alpha, \nu \longleftrightarrow \mu]\}, \\
& \Pi_{\mu \nu, \alpha \beta}^{f_{2}\left(a_{2}\right)}(q, T) \\
& =-\frac{i}{8} \int d^{4} x e^{i q \cdot(x-y)} \\
& \cdot\left\{\operatorname { T r } \left[S_{u}(y-x) \gamma_{\mu} \stackrel{\leftrightarrow}{\mathscr{D}}_{\nu}(x) \stackrel{\leftrightarrow}{\mathscr{D}}_{\beta}(y) S_{u}(x-y) \gamma_{\alpha}\right.\right. \\
& \left.+S_{d}(y-x) \gamma_{\mu} \stackrel{\leftrightarrow}{D}_{\nu}(x) \stackrel{\leftrightarrow}{\mathscr{D}}_{\beta}(y) S_{d}(x-y) \gamma_{\alpha}\right]
\end{aligned}
$$

$$
\begin{aligned}
& +[\beta \longleftrightarrow \alpha]+[\nu \longleftrightarrow \mu] \\
& +[\beta \longleftrightarrow \alpha, \nu \longleftrightarrow \mu]\} .
\end{aligned}
$$

To proceed, we need to know the thermal light quark propagator $S_{q=u, d, s}(x-y)$ in coordinate space which is given as $[18,23]$

$$
\begin{aligned}
S_{q}^{i j}(x-y)= & i \frac{\not x-\not y}{2 \pi^{2}(x-y)^{4}} \delta_{i j}-\frac{m_{q}}{4 \pi^{2}(x-y)^{2}} \delta_{i j}-\frac{\langle\bar{q} q\rangle}{12} \delta_{i j} \\
& -\frac{(x-y)^{2}}{192} m_{0}^{2}\langle\bar{q} q\rangle\left[1-i \frac{m_{q}}{6}(\not x-\not y)\right] \delta_{i j} \\
& +\frac{i}{3}\left[(\not x-\not y)\left(\frac{m_{q}}{16}\langle\bar{q} q\rangle-\frac{1}{12}\left\langle u \Theta^{f} u\right\rangle\right)\right. \\
& \left.+\frac{1}{3}\left(u \cdot(x-y) \not u\left\langle u \Theta^{f} u\right\rangle\right)\right] \delta_{i j} \\
& \cdot\left(\left(\not x-\not g_{s}(x-y)^{2} \sigma_{\mu \nu}^{\mu \nu}+\sigma^{\mu \nu}(\not x-\not y)\right) \delta_{i j},\right.
\end{aligned}
$$

where $\langle\bar{q} q\rangle$ is the temperature-dependent quark condensate, $\Theta_{\mu \nu}^{f}$ is the fermionic part of the energy momentum tensor, and $u_{\mu}$ is the four-velocity vector of the heat bath. In the rest frame of the heat bath, $u_{\mu}=(1,0,0,0)$ and $u^{2}=1$.

The next step is to use the expressions of the propagators and apply the derivatives with respect to $x$ and $y$ in (13). After lengthy but straightforward calculations, the spectral densities at different channels are obtained as

$$
\begin{gathered}
\rho_{K_{2}^{*}}(s)=N_{c}\left(\frac{m_{d} m_{s} s}{32 \pi^{2}}+\frac{s^{2}}{160 \pi^{2}}\right), \\
\rho_{f_{2}\left(a_{2}\right)}(s)=N_{c}\left(\frac{\left(m_{u}^{2}+m_{d}^{2}\right) s}{96 \pi^{2}}+\frac{s^{2}}{160 \pi^{2}}\right),
\end{gathered}
$$

where $N_{c}=3$ is the number of colors. From a similar way, for the nonperturbative contributions, we get

$$
\begin{aligned}
\Pi_{K_{2}^{*}}^{\text {non-pert }}(q, T)= & \frac{\left(6 m_{s}-5 m_{d}\right) m_{0}^{2}}{144 q^{2}}\langle\bar{d} d\rangle \\
& +\frac{\left(6 m_{d}-5 m_{s}\right) m_{0}^{2}}{144 q^{2}}\langle\bar{s} s\rangle \\
& -\frac{2\left\langle u \Theta^{f} u\right\rangle(q \cdot u)^{2}}{9 q^{2}}, \\
\Pi_{f_{2}\left(a_{2}\right)}^{\text {non-pert }}(q, T)= & \frac{m_{d} m_{0}^{2}}{144 q^{2}}\langle\bar{d} d\rangle+\frac{m_{u} m_{0}^{2}}{144 q^{2}}\langle\bar{u} u\rangle \\
& -\frac{2\left\langle u \Theta^{f} u\right\rangle(q \cdot u)^{2}}{9 q^{2}} .
\end{aligned}
$$




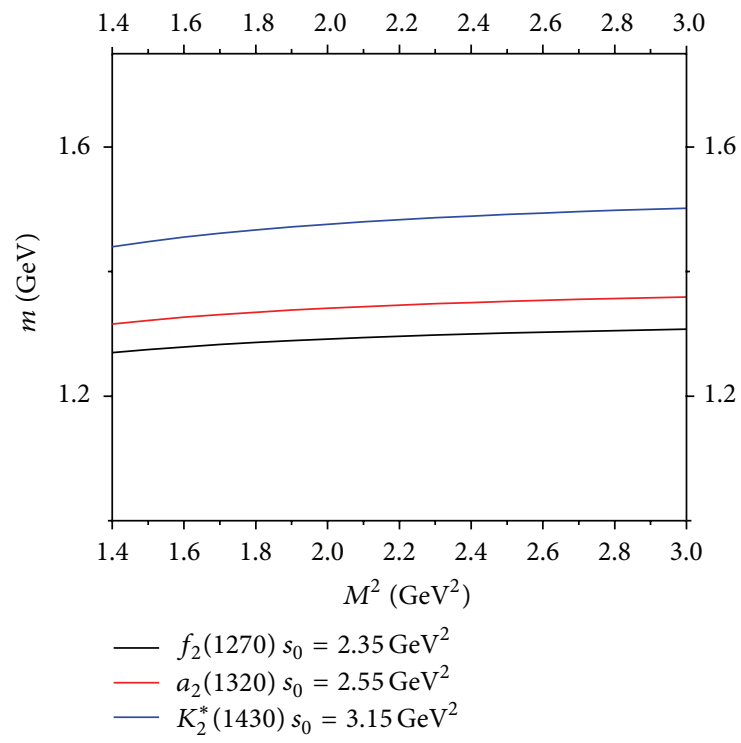

(a)

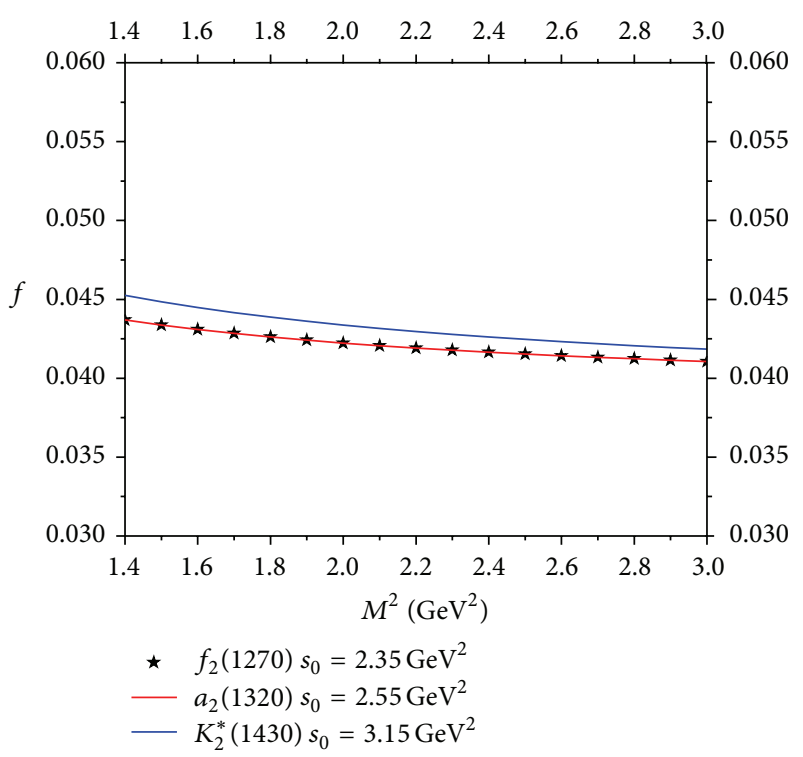

(b)

Figure 1: Variations of the masses and decay constants of the $K_{2}^{*}(1430), f_{2}(1270)$, and $a_{2}(1320)$ mesons with respect to $M^{2}$ at fixed values of the continuum threshold and at zero temperature.

After matching the hadronic and OPE representations, applying Borel transformation with respect to $q^{2}$, and performing continuum subtraction, we obtain the following temperature-dependent sum rule:

$$
\begin{aligned}
f_{K_{2}^{*}\left(f_{2}\right)\left(a_{2}\right)}^{2}(T) m_{K_{2}^{*}\left(f_{2}\right)\left(a_{2}\right)}^{6}(T) \exp \left[\frac{-m_{K_{2}^{*}\left(f_{2}\right)\left(a_{2}\right)}^{2}(T)}{M^{2}}\right] \\
=\int_{\left(m_{q}+m_{d}\right)^{2}}^{s_{0}(T)} d s\left\{\rho_{K_{2}^{*}\left(f_{2}\right)\left(a_{2}\right)}(s) \exp \left[\frac{-s}{M^{2}}\right]\right\} \\
+\widehat{\mathscr{B}} \Pi_{K_{2}^{*}\left(f_{2}\right)\left(a_{2}\right)}^{\text {non-pert }}(q, T),
\end{aligned}
$$

where $\widehat{\mathscr{B}}$ denotes the Borel transformation with respect to $q^{2}, M^{2}$ is the Borel mass parameter, $s_{0}(T)$ is the temperaturedependent continuum threshold, and $m_{q}$ can be $m_{u}, m_{d}$, or $m_{s}$ depending on the kind of tensor meson. The temperaturedependent mass of the considered tensor states is found as

$$
\begin{aligned}
m_{K_{2}^{*}\left(f_{2}\right)\left(a_{2}\right)}^{2}(T) & \\
= & \left(\int_{\left(m_{q}+m_{d}\right)^{2}}^{s_{0}(T)} d s\left\{\rho_{K_{2}^{*}\left(f_{2}\right)\left(a_{2}\right)}(s) s \exp \left[\frac{-s}{M^{2}}\right]\right\}\right. \\
& \left.-\frac{d}{d\left(1 / M^{2}\right)}\left[\widehat{\mathscr{B}} \Pi_{K_{2}^{*}\left(f_{2}\right)\left(a_{2}\right)}^{\text {non-pert }}\right]\right) \\
& \cdot\left(\int_{\left(m_{q}+m_{d}\right)^{2}}^{s_{0}(T)} d s\left\{\rho_{K_{2}^{*}\left(f_{2}\right)\left(a_{2}\right)}(s) \exp \left[\frac{-s}{M^{2}}\right]\right\}\right. \\
& \left.+\widehat{\mathscr{B}} \Pi_{K_{2}^{*}\left(f_{2}\right)\left(a_{2}\right)}^{\text {non-pert }}\right)^{-1} .
\end{aligned}
$$

\section{Numerical Analysis}

In this section, we discuss the sensitivity of the masses and decay constants of the $f_{2}, a_{2}$, and $K_{2}^{*}$ tensor mesons to temperature and compare the results obtained at zero temperature with the predictions of vacuum sum rules [19, 21] as well as the existing experimental data [22]. For this aim, we use some input parameters as $m_{u}=\left(2.3_{-0.5}^{+0.7}\right) \mathrm{MeV}$, $m_{d}=\left(4.8_{-0.3}^{+0.7}\right) \mathrm{MeV}$, and $m_{s}=(95 \pm 5) \mathrm{MeV}$ [22] and $\langle 0|\bar{u} u| 0\rangle=\langle 0|\bar{d} d| 0\rangle=-(0.24 \pm 0.01)^{3} \mathrm{GeV}^{3}$ [24] and $\langle 0|\bar{s} s| 0\rangle=0.8\langle 0|\bar{u} u| 0\rangle[25]$.

In further analysis, we need to know the expression of the light quark condensate at finite temperature calculated at different works (see, e.g., $[26,27]$ ). In the present study, we use the parametrization obtained in [27] which is also consistent with the lattice results [28, 29]. For the temperature-dependent continuum threshold, we also use the parametrization obtained in [27] in terms of the temperature-dependent light quark condensate and continuum threshold in vacuum $\left(s_{0}\right)$. The continuum threshold $s_{0}$ is not completely arbitrary and is correlated with the energy of the first excited state with the same quantum numbers as the chosen interpolating currents. Our analysis reveals that, in the intervals (2.2-2.5) $\mathrm{GeV}^{2},(2.4-2.7) \mathrm{GeV}^{2}$, and (3.0-3.3) $\mathrm{GeV}^{2}$, respectively, for $f_{2}, a_{2}$, and $K_{2}^{*}$ channels, the results weakly depend on the continuum threshold. Hence, we consider these intervals as working regions of $s_{0}$ for the channels under consideration.

According to the general philosophy of the method used, the physical quantities under consideration should also be practically independent of the Borel mass parameter $M^{2}$. The working regions for this parameter are determined by requiring that not only are the higher state and continuum 


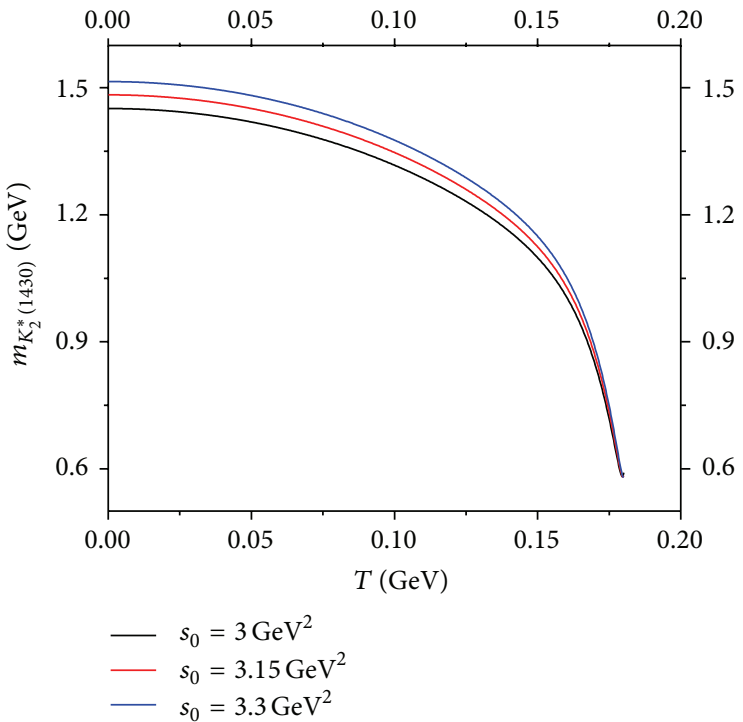

(a)

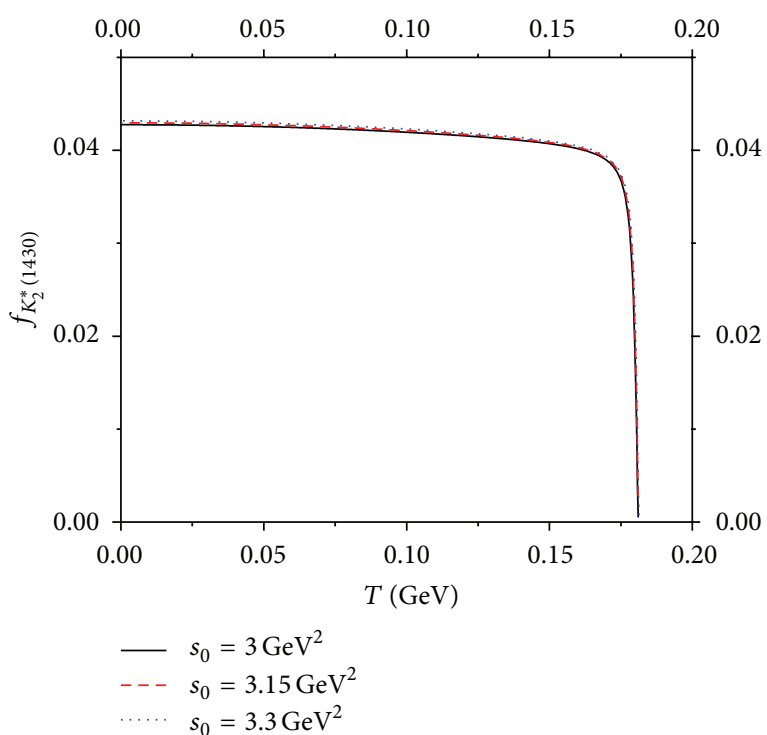

(b)

Figure 2: Temperature dependence of the mass and decay constant of the $K_{2}^{*}(1430)$ meson.

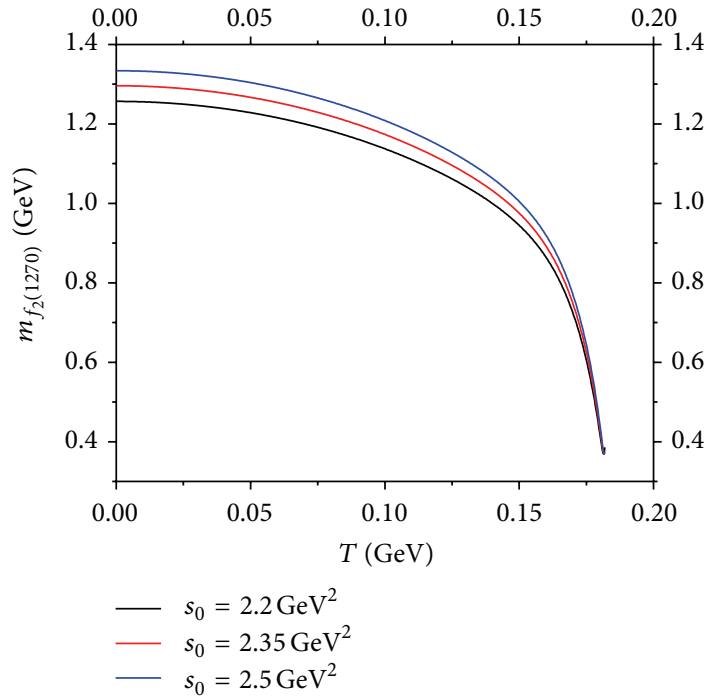

(a)

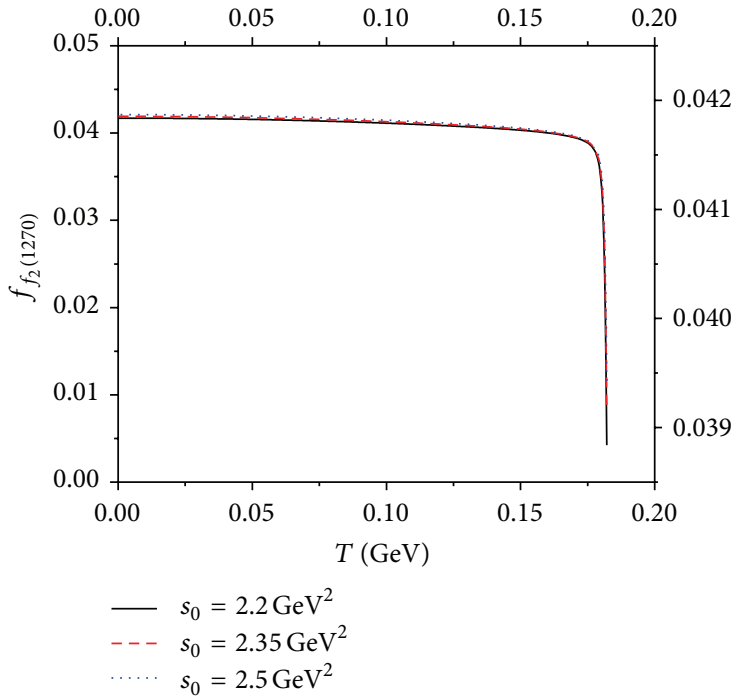

(b)

FIGURE 3: Temperature dependence of the mass and decay constant of the $f_{2}(1270)$ meson.

contributions suppressed, but also the contributions of the highest order operator are small. Taking into account these conditions, we find that, in the interval $1.4 \mathrm{GeV}^{2} \leq M^{2} \leq$ $3 \mathrm{GeV}^{2}$, the results weakly depend on $M^{2}$. Figure 1 indicates the dependence of the masses and decay constants on the Borel mass parameter at zero temperature. From this figure, we see that the results demonstrate good stability with respect to the variations of $M^{2}$ in its working region.

Now, we proceed to discuss how the physical quantities under consideration behave in terms of temperature in the working regions of the auxiliary parameters $M^{2}$ and $s_{0}$. For this aim, we present the dependence of the masses and decay constants on temperature at $M^{2}=2.2 \mathrm{GeV}^{2}$ in Figures 2, 3, and 4. Note that we plot these figures up to the temperature at which the temperature-dependent continuum threshold vanishes; that is, $T \simeq 183 \mathrm{MeV}$. From these figures, we see that the masses and decay constants diminish by increasing the temperature. Near to the temperature $T \simeq 183 \mathrm{MeV}$, the decay constants of the $f_{2}(1270), a_{2}(1320)$, and $K_{2}^{*}(1430)$ decrease with amount of $81 \%, 70 \%$, and $85 \%$ compared to their vacuum values, respectively, while the masses decrease about $70 \%, 72 \%$, and $60 \%$ for $f_{2}(1270), a_{2}(1320)$, and $K_{2}^{*}(1430)$ states, respectively. 


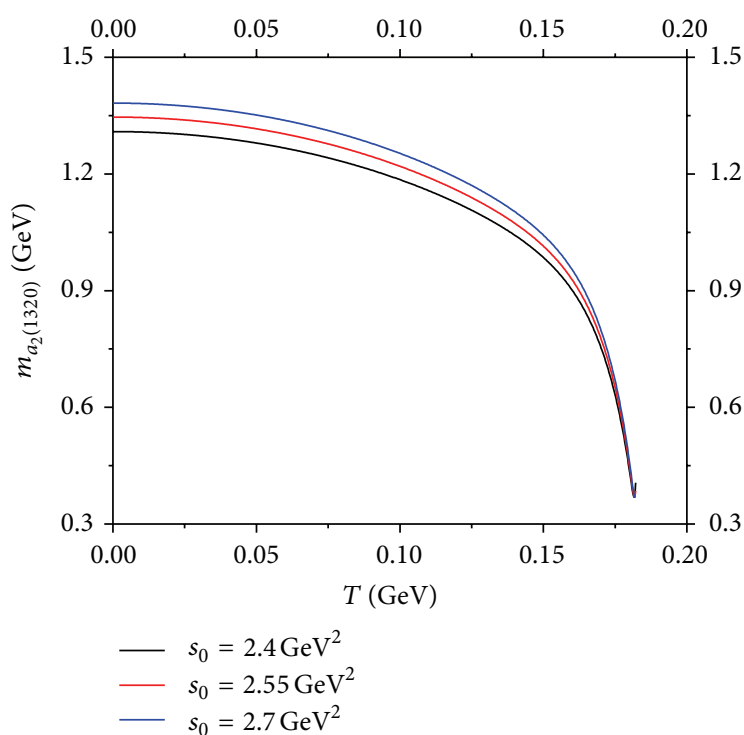

(a)

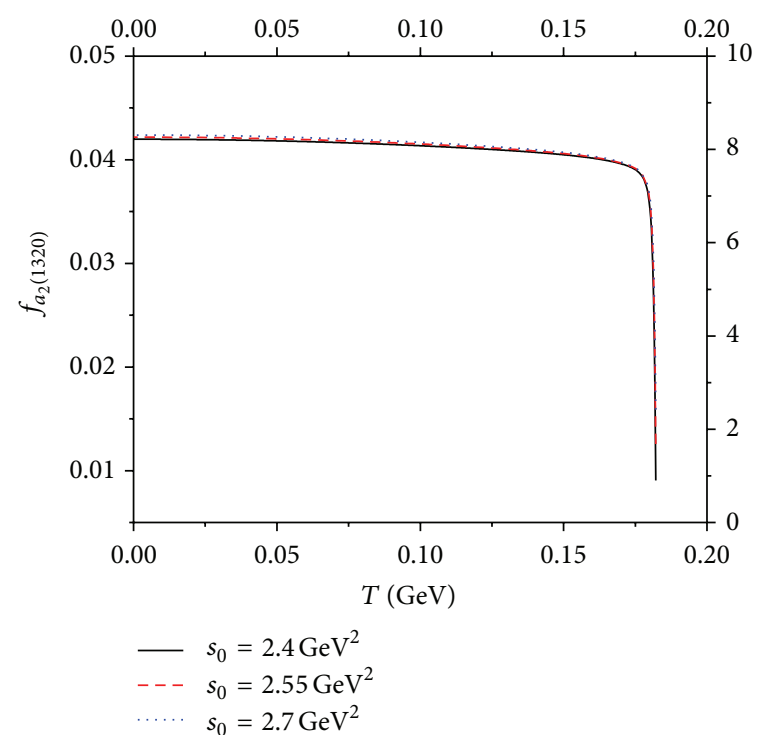

(b)

FIgURE 4: Temperature dependence of the mass and decay constant of the $a_{2}(1320)$ meson.

TABLE 1: Values of the masses and decay constants of the $K_{2}^{*}, f_{2}$, and $a_{2}$ mesons at zero temperature.

\begin{tabular}{lccc}
\hline & Present work & Experiment [22] & $\begin{array}{c}\text { Vacuum sum rules [19, 20], } \\
\text { relativistic quark model [20] }\end{array}$ \\
\hline$m_{K_{2}^{*}(1430)}(\mathrm{GeV})$ & $1.48 \pm 0.12$ & $1.4256 \pm 0.0015$ & $1.44 \pm 0.10[21], 1.424[20]$ \\
$f_{K_{2}^{*}(1430)}(\mathrm{GeV})$ & $0.043 \pm 0.002$ & - & $0.050 \pm 0.002[21]$ \\
$m_{f_{2}(1270)}$ & $1.30 \pm 0.08$ & $1.2751 \pm 0.0012$ & $1.25[19]$ \\
$f_{f_{2}(1270)}(\mathrm{GeV})$ & $0.042 \pm 0.002$ & - & $0.040[19]$ \\
$m_{a_{2}(1320)}$ & $1.35 \pm 0.11$ & - & $1.25[19]$ \\
$f_{a_{2}(1320)}$ & $0.042 \pm 0.002$ & - \\
\hline
\end{tabular}

Our final task is to compare the results of this work obtained at zero temperature with those of the vacuum sum rules as well as other existing theoretical predictions and experimental data. This comparison is made in Table 1. From this table, we see that the results on the masses and decay constants obtained at zero temperature are roughly consistent with existing experimental data as well as the vacuum sum rules and relativistic quark model predictions within the uncertainties. Our predictions on the decay constants of the light tensor mesons can be checked in future experiments. The results obtained in the present work can be used in theoretical determination of the electromagnetic properties of the light tensor mesons as well as their weak decay parameters and their strong couplings with other hadrons. Our results on the thermal behavior of the masses and decay constants can also be useful in analysis of the results of future heavy-ion collision experiments.

\section{Conflict of Interests}

The authors declare that there is no conflict of interests regarding the publication of this paper.

\section{Acknowledgment}

This work has been supported in part by the Scientific and Technological Research Council of Turkey (TUBITAK) under the Research Projects 110T284 and 114F018.

\section{References}

[1] G. E. Brown and M. Rho, "Chiral restoration in hot and/or dense matter," Physics Reports, vol. 269, no. 6, pp. 333-380, 1996.

[2] M. A. Shifman, A. I. Vainshtein, and V. I. Zakharov, "QCD and resonance physics. theoretical foundations," Nuclear Physics, Section B, vol. 147, no. 5, pp. 385-447, 1979.

[3] M. A. Shifman, A. I. Vainshtein, and V. I. Zakharov, "QCD and resonance physics. Applications," Nuclear Physics B, vol. 147, no. 5, pp. 448-518, 1979.

[4] A. I. Bochkarev and M. E. Shaposhnikov, "The spectrum of hot hadronic matter and finite-temperature QCD sum rules," Nuclear Physics B, vol. 268, no. 1, pp. 220-252, 1986.

[5] T. Hatsuda, Y. Koike, and S. H. Lee, "Finite-temperature QCD sum rules reexamined: $\varrho, \omega$ and $\mathrm{A}_{1}$ mesons," Nuclear Physics $B$, vol. 394, no. 1, pp. 221-264, 1993. 
[6] S. Mallik, "Operator product expansion at finite temperature," Physics Letters B, vol. 416, no. 3-4, pp. 373-378, 1998.

[7] E. V. Shuryak, "Correlation functions in the QCD vacuum," Reviews of Modern Physics, vol. 65, no. 1, pp. 1-46, 1993.

[8] S. Mallik and S. Sarkar, "Vector and axial-vector mesons at finite temperature," The European Physical Journal C-Particles and Fields, vol. 25, no. 3, pp. 445-452, 2002.

[9] T. Waas, N. Kaiser, and W. Weise, "Low energy $\overline{\mathrm{K}} \mathrm{N}$ interaction in nuclear matter," Physics Letters B, vol. 365, pp. 12-16, 1996.

[10] L. Tolos, D. Cabrera, and A. Ramos, "Strange mesons in nuclear matter at finite temperature," Physical Review C, vol. 78, Article ID 045205, 2008.

[11] E. V. Veliev, "Operator product expansion for the thermal correlator of scalar currents," Journal of Physics G: Nuclear and Particle Physics, vol. 35, Article ID 035004, 2008.

[12] E. V. Veliev, K. Azizi, H. Sundu, and N. Akşit, "Investigation of heavy-heavy pseudoscalar mesons in thermal QCD sum rules," Journal of Physics G, vol. 39, no. 1, Article ID 015002, 2012.

[13] E. V. Veliev and G. Kaya, "Leptonic decay constants of $D_{s}$ and $B_{s}$ mesons at finite temperature," The European Physical Journal C, vol. 63, no. 1, pp. 87-91, 2009.

[14] F. Klingl, S. Kim, S. H. Lee, P. Morath, and W. Weise, "Masses of $J / \psi$ and $\eta c$ in the nuclear medium: QCD sum rule approach," Physical Review Letters, vol. 82, no. 17, pp. 3396-3399, 1999.

[15] C. A. Dominguez, M. Loewe, and J. C. Rojas, "Heavy-light quark pseudoscalar and vector mesons at finite temperature," Journal of High Energy Physics, vol. 2007, no. 8, article 040, 2007.

[16] C. A. Dominguez and M. Loewe, "Deconfinement and chiralsymmetry restoration at finite temperature," Physics Letters $B$, vol. 233, pp. 201-204, 1989.

[17] E. V. Veliev, K. Azizi, H. Sundu, G. Kaya, and A. Türkan, "Thermal qcd sum rules study of vector charmonium and bottomonium states," The European Physical Journal A, vol. 47, no. 9, p. 110, 2011.

[18] K. Azizi, H. Sundu, A. Türkan, and E. V. Veliev, “Thermal properties of $D_{s 2}^{*}(2460)$ and $D_{s 2}^{*}(2573)$ tensor mesons using QCD sum rules," Journal of Physics G: Nuclear and Particle Physics, vol. 41, no. 3, Article ID 035003, 2014.

[19] T. M. Aliev and M. A. Shifman, "Old tensor mesons in QCD sum rules,” Physics Letters B, vol. 112, pp. 401-405, 1982.

[20] D. Ebert, R. N. Faustov, and V. O. Galkin, "Mass spectra and Regge trajectories of light mesons in the relativistic quark model," Physical Review D, vol. 79, no. 11, Article ID 114029, 11 pages, 2009.

[21] T. M. Aliev, K. Azizi, and V. Bashiry, "On the mass and decay constant of $\mathrm{K}_{2}^{*}(1430)$ tensor meson," Journal of Physics G: Nuclear and Particle Physics, vol. 37, no. 2, Article ID 025001, 2010.

[22] J. Beringer, J. F. Arguin, R. M. Barnett et al., "Review of particle physics," Physical Review D, vol. 86, Article ID 010001, 2012.

[23] Z.-G. Wang, Z.-C. Liu, and X.-H. Zhang, "Analysis of the $Y(4140)$ and related molecular states with QCD sum rules," The European Physical Journal C, vol. 64, no. 3, pp. 373-386, 2009.

[24] B. L. Ioffe, "QCD (Quantum chromodynamics) at low energies," Progress in Particle and Nuclear Physics, vol. 56, pp. 232-277, 2006.

[25] S. Narison, "Open charm and beauty chiral multiplets in QCD," Physics Letters B, vol. 605, no. 3-4, pp. 319-325, 2005.

[26] A. Barducci, R. Casalbuoni, S. de Curtis, R. Gatto, and G. Pettini, "Current quark mass and chiral-symmetry breaking in QCD at finite temperature in a mean-field approximation," Physical Review D, vol. 46, no. 5, pp. 2203-2211, 1992.

[27] A. Ayala, A. Bashir, C. A. Dominguez, E. Gutiérrez, M. Loewe, and A. Raya, "QCD phase diagram from finite energy sum rules," Physical Review D, vol. 84, Article ID 056004, 2011.

[28] A. Bazavov, T. Bhattacharya, M. Cheng et al., "Equation of state and QCD transition at finite temperature," Physical Review D, vol. 80, Article ID 014504, 2009.

[29] M. Cheng, S. Ejiri, P. Hegde et al., "Equation of state for physical quark masses," Physical Review D, vol. 81, no. 5, Article ID 054504, 8 pages, 2010. 

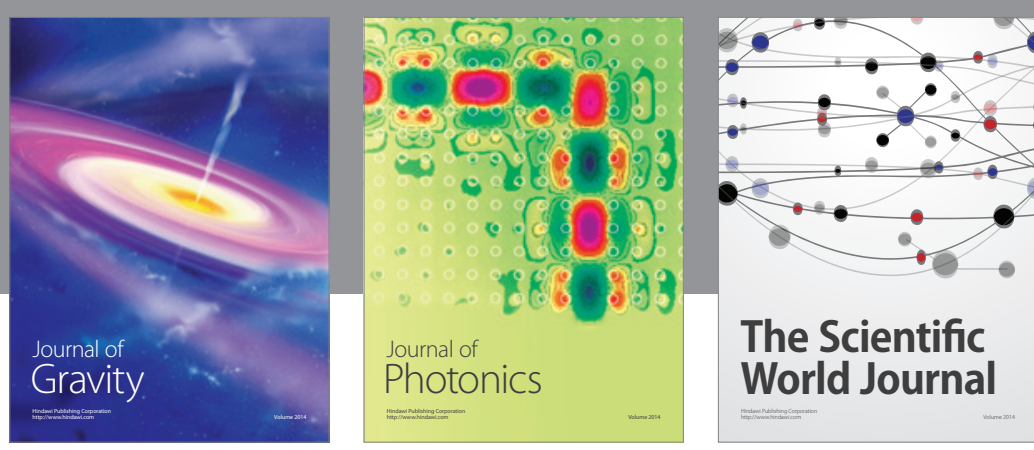

The Scientific World Journal
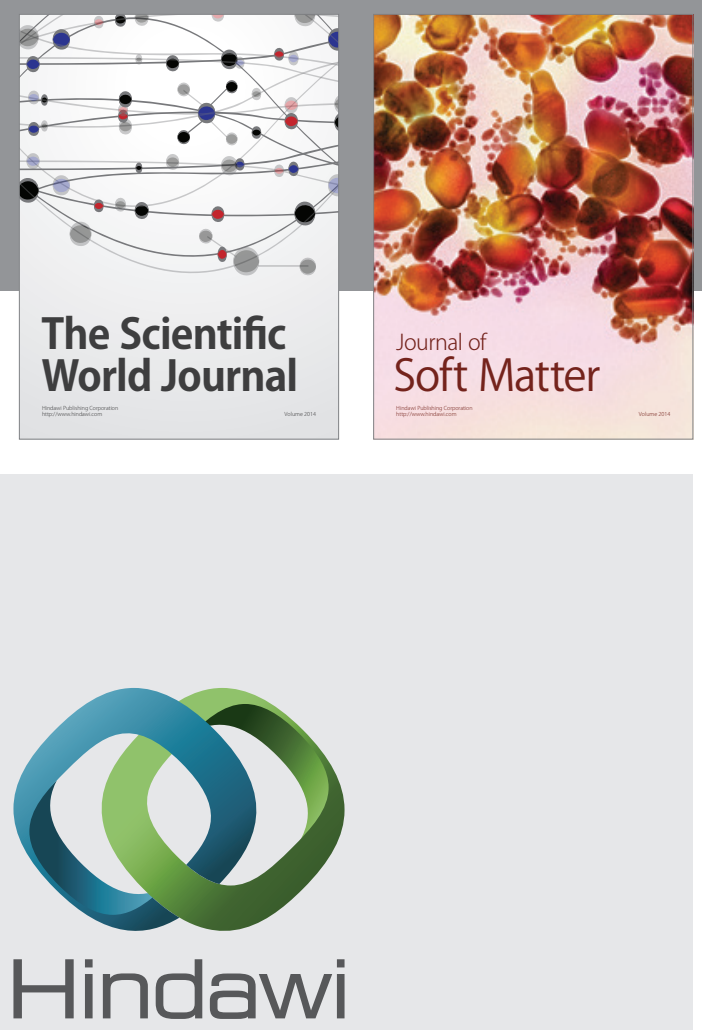

Submit your manuscripts at

http://www.hindawi.com

nternational Journal of

Statistical Mechanics
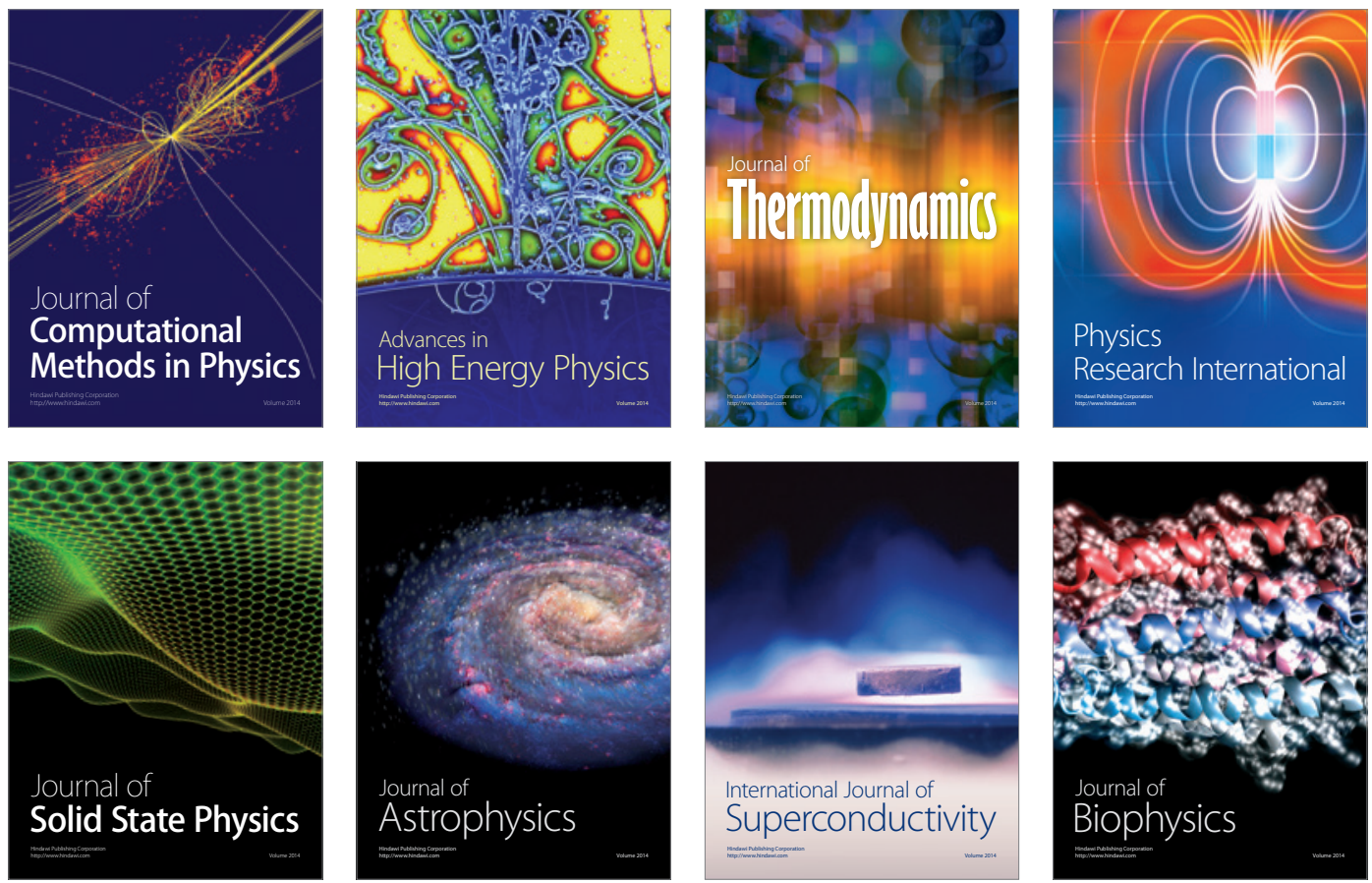
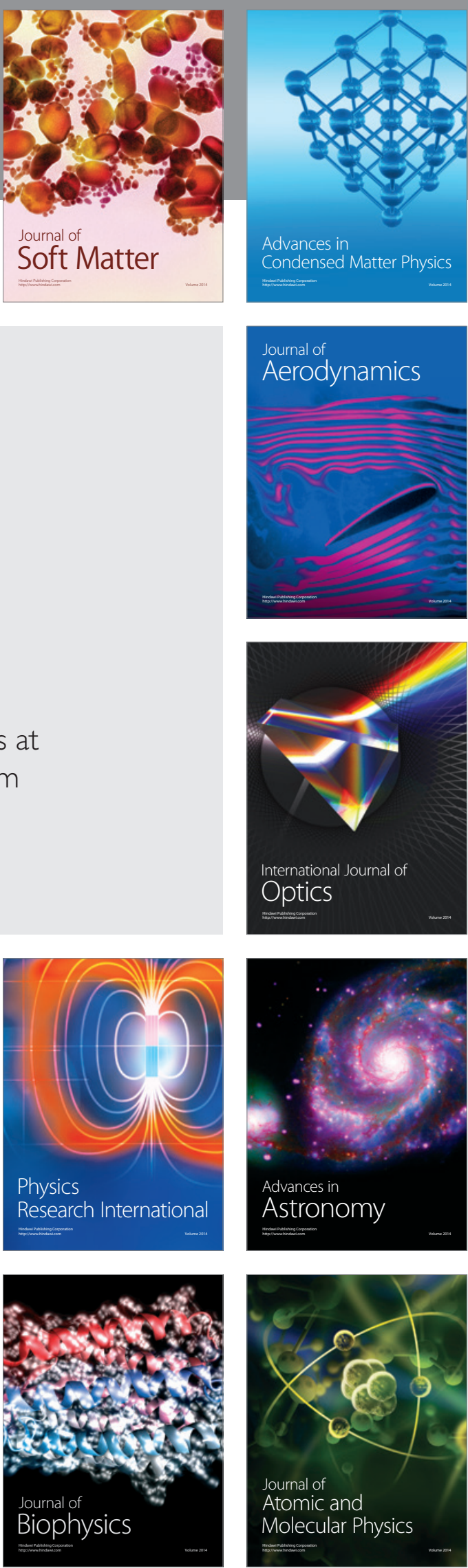